\title{
Effect of Vitiligo on Auditory Functions: Is There Any Association?
}

\author{
Vishal Singh ${ }^{1} \quad$ Trilok C. Guleria ${ }^{1} \quad$ Ramesh K. Azad $^{1} \quad$ Narender K. Mohindroo ${ }^{1} \quad$ Devraj Sharma ${ }^{1}$ \\ ${ }^{1}$ Department of ENT, Indira Gandhi Medical College, Shimla, \\ Address for correspondence Trilok C. Guleria, MBBS, MS, Department \\ Himachal Pradesh, India \\ of ENT, Indira Gandhi Medical College, Circular Road, Lakkar Bazar, \\ Int Arch Otorhinolaryngol 2021;25(2):e200-e204. \\ Shimla, HP, 171001, India (e-mail: tcguleria@gmail.com).
}

\section{Abstract}

Keywords

- vitiligo

- stria vascularis

- nonsegmental

- audiometric

- auditory defect

Introduction Vitiligo is an acquired pigmentary disorder of unknown etiology, clinically characterized by whitish macules caused by selective loss of melanocytes. There are many melanocytes in the human cochlea. Recent studies have suggested a direct relation between cochlear dysfunction and decreased amounts of melanin.

Objective To determine the effect of vitiligo on auditory function.

Methods The present prospective, case control study was performed over a period of 1 year in patients between 15 and 40 years old with nonsegmental disease and the same number of controls. Pure tone audiometry (PTA) and Otoacoustic emission (OAE) were performed to analyze the correlation between vitiligo and auditory function.

Results The mean pure tone audiometric threshold in the right ear at $0.5,8 \mathrm{kHz}$, and in the left ear at the frequency of $8 \mathrm{kHz}$ in the case group were significantly higher as compared with controls. The average band reproducibility (\%) in the right and left ear of controls was significantly higher at the frequency of $4 \mathrm{kHz}$ as compared with cases. It has been observed that signal to noise ratio was statistically higher at the frequency of $5 \mathrm{kHz}$ in the right ear and at 2,3 , and $5 \mathrm{kHz}$ in the left ear in controls as compared with cases. On the basis of disease type and duration of disease; mean pure tone audiometric threshold, average band reproducibility and signal to noise ratio, no significant effect was observed in the present study.

Conclusion Vitiligo has an effect on cochlear function irrespective of the duration or distribution of the disease.
\end{abstract}

\section{Introduction}

Vitiligo is an acquired pigmentary disorder of unknown etiology, clinically characterized by whitish macules caused by selective loss of melanocytes. ${ }^{1}$ Vitiligo has been reported in $50 \%$ of cases before 20 years old; however, the late onset of the disease may be associated with other diseases like rheumatoid arthritis and diabetes mellitus (DM). Females usually acquire the disease earlier, that is, in the $1^{\text {st }}$ decade; in the case of males, the peak of the disease is in the $5^{\text {th }}$ decade. The disease appears to be transmitted in a multifactorial manner and lesions typically develop in areas of friction., ${ }^{2,3}$ Vitiligo may be classified as localized, generalized and universal, according to the area of pigmentation loss. Generalized vitiligo is the most common type and vitiligo vulgaris is the most common subtype. Based on its etiopathogenesis, vitiligo has been classified as immune (progressive vitiligo)/nonsegmental, neural (segmental/dermatomal), and chemical (contact). ${ }^{4,5}$

Embryonically, melanocytes develop from the neural crest, later on distributed in the epidermis of hair bulbs in received

May 30, 2019

accepted

February 25, 2020

published online

June 23, 2020
DOI https://doi.org/ 10.1055/s-0040-1709197. ISSN 1809-9777.

\footnotetext{
(c) 2020. Fundação Otorrinolaringologia. All rights reserved.

This is an open access article published by Thieme under the terms of the Creative Commons Attribution-NonDerivative-NonCommercial-License, permitting copying and reproduction so long as the original work is given appropriate credit. Contents may not be used for commercial purposes, or adapted, remixed, transformed or built upon. (https://creativecommons.org/ licenses/by-nc-nd/4.0/) Thieme Revinter Publicações Ltda., Rua do Matoso 170, Rio de Janeiro, RJ, CEP 20270-135, Brazil
} 
the skin, the uveal tract, the retinal pigment epithelium, the inner ear and the leptomeninges, which are collectively regarded as melanocyte organs. ${ }^{6}$ Melanocytes appear to be vital for the normal development and function of the stria vascularis. Melanocytes may have some direct, essential role in the production of electrocochlear potential by the stria. ${ }^{7}$ There are many melanocytes in the human cochlea, particularly in the modiolus, in the osseous spiral lamina, in the Reissner membrane, and in the vascular stria. Melanin may have an important role in the inner ear, since hearing is affected in systemic disorders that affect pigmented areas (eyes, skin, and hair) such as Vogt-Koyanagi Harada (VKH) and Waardenburg syndromes. ${ }^{8}$ Although the loss or reduction of melanocytes in the inner ear may have a critical effect on hearing, if it affects the perception of the frequencies of the sound used in normal verbal communication or only the higher frequencies, and how duration of the disease affects normal hearing is still a matter of debate.

Therefore, we conducted a prospective, case control study to determine the effect of vitiligo as well as of the duration and type of vitiligo on hearing.

\section{Materials and Methods}

After institutional ethical board approval, 40 vitiligo patients with nonsegmental disease and 40 age-matched healthy controls were enrolled over a period of 1 year after signing a written informed consent. Subjects with ear pathology, history of acoustic/noise trauma, systemic disease (i.e., renal insufficiency, DM, hypertension, hypercholesterolemia/dyslipidemia), family history of hereditary hearing loss, taking ototoxic drugs or steroids, smokers and having other dermatological disorders were excluded from the study.

The cases and controls underwent a battery of audiological tests. PTA was performed in all of the subjects with an Interacoustics AC 40 Clinical Audiometer (Interacoustics A/S, Assens, Denmark) in a fully sound attenuated room. Hearing loss was considered if the hearing threshold exceeded $25 \mathrm{~dB}$.
OAE testing was done with a GSI audioscreener ${ }^{+}$(VIASYS NeuroCare, Madison, WI, USA) to assess distortion product otoacoustic emissions (DPOAEs) and transient evoked otoacoustic emissions (TEOAEs). Total Band Reproducibility (TBR) in TEOAEs and Signal to Noise Ratio (SNR) in DPOAEs were considered to analyze the results. A pass or refer result for each frequency band is based first on the presence or absence of a DPOAE and TEOAE and second on the absolute level of the DPOAEs and TEOAEs in dB SPL.

Using the methodology described above, the role of PTA and of OAE in knowing the correlation between vitiligo and hearing and its effect on cochlear function was analyzed. All of the values were expressed as mean \pm standard deviation (SD), and significant differences between the case and control groups were determined using the Student $t$-test, with a $p$-value $\leq 0.05$ as cutoff for statistical significance.

\section{Observation and Results}

The present study was conducted in 40 vitiligo patients and 40 healthy controls, with a mean age of 27.65 years old in controls and of 27.25 years old in cases. In the control group, there were 16 males (40\%) and 24 females (60\%), while among cases there were 14 males (35\%) and 26 (65\%) females.

The mean pure tone audiometric threshold in the right ear at the frequencies of 0.5 and $8 \mathrm{kHz}$ in the case group were significantly higher $(p \leq 0.05)$ as compared with controls. Pure tone audiometric average in the left ear in the cases as compared with controls at the frequency of $8 \mathrm{KHz}$ was statistically high $(p \leq 0.05)$. ( - Table 1 )

Transient evoked otoacoustic emissions testing was done in both groups at different frequencies. In the right ear in controls and cases at frequencies of 1,2 , and $4 \mathrm{kHz}$, there were $57.50 \%, 60 \%, 40 \%$ and $17.50 \%, 62.50 \%$, and $5 \%$ pass, respectively; however, refer were $42.50 \%, 40 \%, 60 \%$ and $82.50 \%, 37.50 \%$, and $95 \%$ respectively, in the control and in the case groups. In the left ear in controls and cases at frequencies of 1,2 , and $4 \mathrm{kHz}$, there were $55 \%, 65 \%, 40 \%$

Table 1 Comparison of pure tone audiometric threshold (mean \pm standard deviation) in controls and cases group

\begin{tabular}{|l|l|l|l|l|l|}
\hline Frequency $(\mathrm{kHz})$ & Control & Cases & T & df & $p$-value \\
\hline Right Ear & & & & & \\
\hline 0.5 & $16.38 \pm 4.38$ & $18.75 \pm 5.16$ & -2.22 & 78 & $0.03^{*}$ \\
\hline 1 & $17.38 \pm 3.92$ & $17.75 \pm 5.42$ & -0.35 & 78 & 0.72 \\
\hline 2 & $15.88 \pm 4.06$ & $17.13 \pm 5.87$ & -1.11 & 78 & 0.03 \\
\hline 4 & $18.63 \pm 5.88$ & $21.00 \pm 10.08$ & -1.29 & 78 & 0.02 \\
\hline 8 & $19.00 \pm 7.78$ & $22.88 \pm 8.23$ & -2.16 & 78 & $0.05^{*}$ \\
\hline Left Ear & & & & & \\
\hline 0.5 & $17.63 \pm 3.75$ & $18.50 \pm 4.11$ & -0.99 & 78 & 0.32 \\
\hline 1 & $16.38 \pm 3.75$ & $17.50 \pm 4.67$ & -1.19 & 78 & 0.24 \\
\hline 2 & $15.38 \pm 4.44$ & $16.88 \pm 5.39$ & -1.36 & 78 & 0.19 \\
\hline 4 & $19.50 \pm 8.38$ & $22.38 \pm 10.25$ & -1.37 & 78 & 0.17 \\
\hline 8 & $19.00 \pm 5.45$ & $22.63 \pm 10.62$ & -1.92 & 78 & $0.05^{*}$ \\
\hline
\end{tabular}

Abbreviations: df, degrees of freedom; T, t-distribution. 


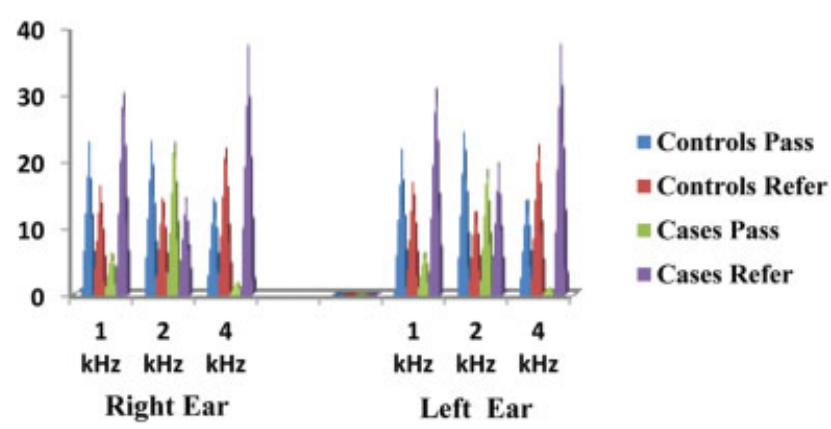

Fig. 1 Comparison of transient evoked otoacoustic emissions results in controls and cases.

and $17.50 \%, 50 \%$, and $2.50 \%$ pass, respectively; however, refer were $45 \%, 35 \%, 60 \%$ and $82.50 \%, 50 \%$, and $97.50 \%$, respectively, in the control and case groups. (-Fig. 1)

It has been observed that average band reproducibility (\%) in controls in the right ear, as well as in left ear, was significantly higher $(p \leq 0.05)$ at the frequency of $4 \mathrm{kHz}$ as compared with cases. (-Table 2 )

Distortion product otoacoustic emissions was done in both groups; among the controls, in the right ear, there were $85 \%$, $97 \%, 92 \%, 90 \%$ and $65 \%$ pass whereas refer were $15 \%, 3 \%, 8 \%$,
$10 \%$ and $35 \%$, respectively, at frequencies of $2,3,4,5$ and $6 \mathrm{kHz}$. In the right ear, $92.50 \%, 85 \%, 82 \%, 75 \%, 65 \%$ pass, and $7.50 \%$, $15 \%, 18 \%, 25 \%, 35 \%$ refer were obtained in cases at the frequencies of $2,3,4,5$, and $6 \mathrm{kHz}$ respectively. The DPOAEs results showed that in the left ear of controls there were $97.50 \%, 92.50 \%, 97.50 \%, 97.50 \%$, and $62.50 \%$ pass and $2.50 \%, 7.50 \%, 2.50 \%, 2.50 \%$, and $37.50 \%$ refer at the frequencies of $2,3,4,5$, and $6 \mathrm{kHz}$, respectively. In cases, $80 \%, 70 \%, 67.50 \%$, $70 \%$, and $50 \%$ pass, and $20 \%, 30 \%, 32.50 \%, 30 \%$, and $50 \%$ refer were obtained at the frequencies of $2,3,4,5$,and $6 \mathrm{kHz}$, respectively. (-Fig. 2 )

Signal to noise ratio was measured at different frequencies, ranging from 2 to $6 \mathrm{kHz}$. We obtained a higher value of SNR in controls as compared with cases, and a statistically higher value $(p \leq 0.05)$ at the frequency of $5 \mathrm{kHz}$ in the right ear and at the frequencies of 2,3 , and $5 \mathrm{kHz}$ in the left ear. ( - Table 3 )

On the basis of type of disease, there were 7 localized and 33 generalized cases of vitiligo. On the basis of duration of disease, they were divided into 2 groups between 1 and 12 years and between 13 and 24 years of duration. The mean pure tone audiometric threshold values were statistically similar $(p>0.05)$ at different frequencies among different types of vitiligo. The average band reproducibility (\%) was not significantly $(p>0.05)$ affected by the different type of vitiligo. It has

Table 2 Comparison of transient evoked otoacoustic emissions band reproducibility (\%; mean \pm standard deviation) in controls and cases

\begin{tabular}{|l|l|l|l|l|l|}
\hline Frequency $(\mathrm{kHz})$ & Control & Cases & T & df & $p$-value \\
\hline Right Ear & & & & & \\
\hline 1 & $48.79 \pm 31.67$ & $40.86 \pm 25.57$ & 1.23 & 78 & 0.22 \\
\hline 2 & $66.99 \pm 29.27$ & $61.56 \pm 30.63$ & -0.81 & 78 & 0.42 \\
\hline 4 & $50.82 \pm 32.60$ & $35.50 \pm 25.95$ & 2.33 & 78 & $0.02^{*}$ \\
\hline Left Ear & & & & & \\
\hline 1 & $47.04 \pm 33.85$ & $44.17 \pm 23.63$ & 0.44 & 78 & 0.66 \\
\hline 2 & $61.40 \pm 32.04$ & $60.97 \pm 29.78$ & 0.06 & 78 & 0.95 \\
\hline 4 & $51.18 \pm 27.99$ & $39.27 \pm 26.38$ & 1.96 & 78 & $0.05^{*}$ \\
\hline
\end{tabular}

Abbreviations: $\mathrm{df}$, degrees of freedom; T, t-distribution.

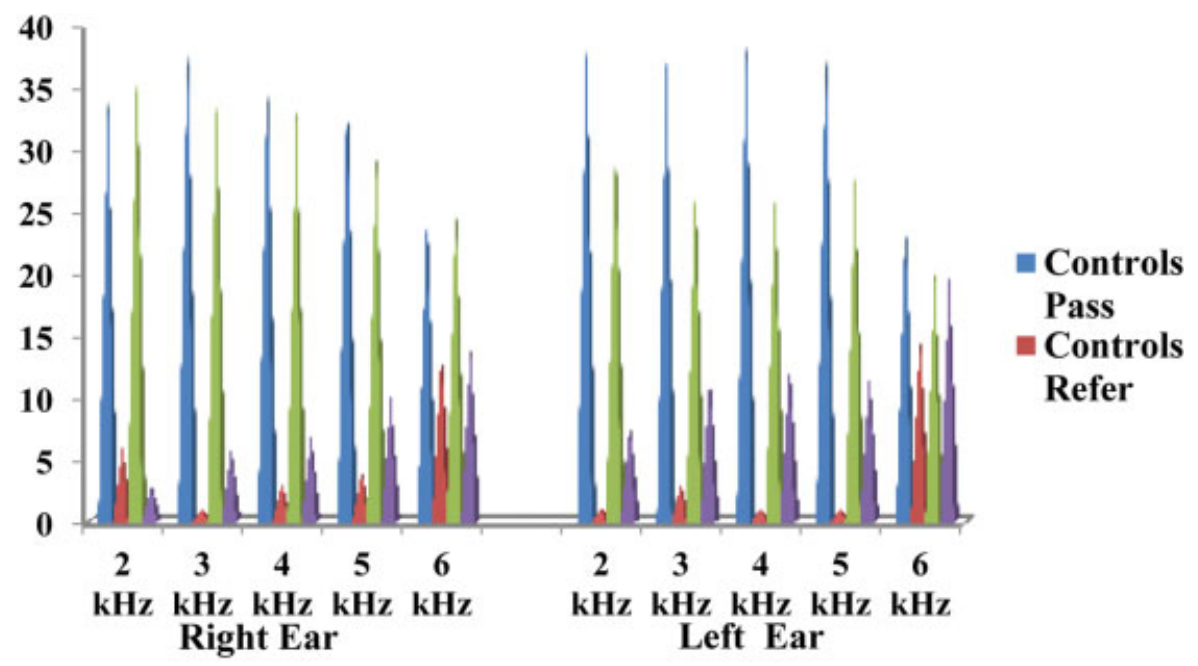

Fig. 2 Comparison of distortion product otoacoustic emissions results in controls and cases. 
Table 3 Comparison of distortion product otoacoustic emissions signal to noise ratio (mean \pm standard deviation) in controls and cases

\begin{tabular}{|c|c|c|c|c|c|}
\hline Frequency (kHz) & Controls & Cases & $\mathrm{T}$ & df & p-value \\
\hline \multicolumn{6}{|l|}{ Right Ear } \\
\hline 2 & $17.29 \pm 8.61$ & $16.56 \pm 9.27$ & 0.37 & 78 & 0.71 \\
\hline 3 & $20.09 \pm 6.76$ & $17.99 \pm 9.36$ & 1.15 & 78 & 0.25 \\
\hline 4 & $22.34 \pm 8.58$ & $20.59 \pm 9.85$ & 0.85 & 78 & 0.39 \\
\hline 5 & $21.17 \pm 8.46$ & $16.65 \pm 10.95$ & 1.15 & 78 & $0.05^{*}$ \\
\hline 6 & $14.68 \pm 11.73$ & $13.07 \pm 10.04$ & 0.66 & 78 & 0.51 \\
\hline \multicolumn{6}{|l|}{ Left Ear } \\
\hline 2 & $18.25 \pm 5.90$ & $14.50 \pm 9.57$ & 2.11 & 78 & $0.04^{*}$ \\
\hline 3 & $19.92 \pm 7.39$ & $15.61 \pm 11.09$ & 2.01 & 78 & $0.05^{*}$ \\
\hline 4 & $21.79 \pm 6.51$ & $18.25 \pm 11.80$ & 1.66 & 78 & 0.10 \\
\hline 5 & $21.24 \pm 6.78$ & $15.93 \pm 11.05$ & 2.59 & 78 & $0.02^{*}$ \\
\hline 6 & $14.55 \pm 12.03$ & $11.30 \pm 10.46$ & 1.29 & 78 & 0.20 \\
\hline
\end{tabular}

Abbreviations: $\mathrm{df}$, degrees of freedom; $\mathrm{T}$, t-distribution.

been observed that there was no statistical difference $(p>0.05)$ between the localized and the generalized type of vitiligo on the mean SNR. The mean pure tone audiometric threshold values were statistically similar $(p>0.05)$ at different frequencies among different durations of vitiligo. The average band reproducibility (\%) was not significantly $(p>0.05)$ affected by the different durations of vitiligo. There was no significant effect $(p>0.05)$ of different durations on DPOAEs signal to noise ratio was observed in the present study.

\section{Discussion}

In the present study, we observed that the pure tone audiometric value in the study group was numerically higher at the frequencies of $1 \mathrm{kHz}, 2 \mathrm{kHz}$, and $4 \mathrm{kHz}$, and statistically higher $(p<0.05)$ at speech $(0.5 \mathrm{kHz})$ and at a higher frequency $(8 \mathrm{kHz})$ as compared with the control group. Thirteen patients (32.5\%) in our study have shown a higher value for PTA. Similarly, Shalaby et al $(2006)^{9}$ stated that the mean of pure tone threshold in the frequencies between 0.25 and $16 \mathrm{KHz}$ was more elevated in the study group than in the control group, but this difference was not statistically significant. The mean pure tone average in the study conducted by Shalaby et al at the frequencies of $1 \mathrm{kHz}$, $2 \mathrm{kHz}, 4 \mathrm{kHz}$, and $8 \mathrm{kHz}$, of cases versus controls, were 21.2 , 21.7, 23.3, 23.3 dB and 18.7, 20.4, 22.3, 20.8 dB, respectively, in the right ear; and in the left ear they were 20.8, 22.9, 23.4, and $22.8 \mathrm{~dB}$ and $18.00,20.8,21.2$, and $19.9 \mathrm{~dB}$, respectively. In our study, the mean pure tone averages at the frequencies of $1 \mathrm{kHz}, 2 \mathrm{kHz}, 4 \mathrm{kHz}$, and $8 \mathrm{kHz}$ were $17.75,17.13,21.00$, and $22.88 \mathrm{~dB}$ and $17.38,15.88,18.63$, and $19.00 \mathrm{~dB}$ in the study and control groups, respectively, in the right ear, and 17.50 and $16.38 \mathrm{~dB}$ at $1 \mathrm{kHz}, 16.88$ and $15.38 \mathrm{~dB}$ at $2 \mathrm{kHz}, 22.38$ and $19.50 \mathrm{~dB}$ at $4 \mathrm{kHz}$ and 22.63 and $19.00 \mathrm{~dB}$ at $8 \mathrm{kHz}$ in the left ear in the study and control groups, respectively. Therefore, we can see that mean pure tone average values in the study group were higher as compared with the control group. Fifteen percent of patients with vitiligo who demonstrated audiologic changes were symptomatically free regarding hearing sensitivity. Like the study conducted by Shalaby et al, in our study none of the patients were symptomatic regarding hearing sensitivity as well. The hearing loss was only subclinical.

Escalante-Ugalde et $\mathrm{al}^{10}$ conducted a case control study taking 100 vitiligo patients and 50 healthy controls. The audiometric threshold between 125 and $8,000 \mathrm{~Hz}$ was normal for both cases and controls. As compared with our study, they found no association between hypoacusis and vitiligo on pure tone audiometry. They said that their patients were nonwhite and different ethnic groups may have different results. We also observed variation for otoacoustic emissions, that is, decreased value of TEOAEs average band reproducibility percentage and DPOAEs SNR in the case group as compared with the control group. The average band reproducibility percentage in all observed frequencies in the study group were less as compared with the control group, and it was statistically significant $(p<0.05)$ at $4 \mathrm{kHz}$ in the right ear $(35.50 \%)$ and in the left ear (39.27\%) in the study group as compared with the control group, that is, $50.82 \%$ and $51.18 \%$, respectively. Eleven patients (27.5\%) have shown failure in TEOAE in both the right and left ears. Similarly, DPOAEs SNR were also decreased in the study group at all frequencies as compared with the control group in the right and left ear, but was significantly lower $(p<0.05)$ at $5 \mathrm{kHz}, 16.65$ in cases and 21.17 in controls in the right ear and 14.50 , and 18.25 at $2 \mathrm{kHz}, 15.61$ and 19.92 at $3 \mathrm{kHz}, 15.93$ and 21.24 at the frequency of $5 \mathrm{kHz}$ in the study and control groups, respectively, in left ear. Ten patients (25\%) had shown absent DPOAE.

Shalaby et $\mathrm{al}^{9}$ revealed a significant decrease in the reproducibility percent of TEOAE in the vitiligo group. The reproducibility percent was $33.3 \%$ in the study group compared with $98 \%$ in the control group. The reproducibility percentage in our study is $35.50 \%$ and $50.82 \%$ in the study and control groups, respectively. 
Angrisani et al $^{11}$ observed that out of 24 patients, 17 (70.89\%) patients had absence of TEOAEs. Similar to this study, in our study TEOAEs were absent in 38 (95\%) in the right ear and in 39 (97.50\%) in the left ear. Distortion product otoacoustic emissions were absent in our study in the right ear in 14(35\%) and in 20 (50\%) in the left ear. Angrisani et al ${ }^{11}$ observed no statistically significant differences in conventional audiometry and otoacoustic emissions when comparing different types and durations of vitiligo. In our study, we found no statistical significant difference in different types of vitiligo in relation to audiological parameters. So the type of distribution of vitiligo patches over the skin may not provide a direct and definite picture of cochlear dysfunction.

Mahdi et al $^{12}$ found no correlation between the duration of the disease and the recorded pure tone audiometric values. Similarly, Sharma et $\mathrm{al}^{13}$ and Elsaied et al ${ }^{14}$ observed that there was no effect of duration on the audiological parameters. In our study, we have found no statistical significant difference in audiological parameters used to assess hearing in different durations of vitiligo. They have explained that it may be possible that otic melanocytes are affected at the start of vitiligo and then stabilize afterwards. ${ }^{9}$

Otoacostic emissions (OAEs) are the result of active processing by outer hair cells. OAEs are present when outer hair cells are healthy and absent outer hair cells are damaged. Thus, OAEs reveal, with exquisite senstivity, the integrity of outer hair cell function. ${ }^{11}$ In the present study, decreased otoacoustic emissions, that is, TEOAEs average band reproducibility percentage and DPOAEs SNR in the case group strengthens the hypothesis that vitiligo is a significant factor for altered cochlear function, and that melanin may in fact have an important role in cell metabolism, facilitating substance exchanges and maintaining the endolymph, perilymph and ionic balance.

In our study, none of the patients was symptomatic regarding hearing sensitivity, and hearing loss was only subclinical. In our study, we found no statistical significant difference in different types of vitiligo and in different durations of vitiligo. However, the small sample size and limited period of study may be taken as limitations of the present study. For a complete evaluation, further studies should include a large sample size and longer duration of the studies.

\section{Conclusion}

Vitiligo has an effect on cochlear function and the affection is usually asymptomatic subclinical hearing loss for a long time. Otoacoustic emissions is a valuable tool in the detection of minimal cochlear lesion. Some patients with vitiligo will have a certain amount of auditory defect irrespective of the duration or distribution of the disease. All vitiligo patients require routine monitoring and audiological assessments for early identification and monitoring of changes as the disease progresses, with pure tone audiometry and OAE. To obtain more precise results about the hearing status of patients with vitiligo, a larger sample size and longer duration of studies are recommended.

\section{Conflict of Interests}

The authors have no conflict of interests to declare.

\section{References}

1 Lerner AB. On the etiology of vitiligo and gray hair. Am J Med 1971;51(02):141-147

2 Howitz J, Brodthagen H, Schwartz M, Thomsen K. Prevalence of vitiligo. Epidemiological survey on the Isle of Bornholm, Denmark. Arch Dermatol 1977;113(01):47-52

3 Woolfson H, Finn OA, Mackie RM, McQueen A, MacSween RNM. Serum anti-tumour antibodies and auto-antibodies in vitiligo. $\mathrm{Br} \mathrm{J}$ Dermatol 1975;92(04):395-400

4 Birbeck MS, Breathnach AS, Everall JD. An electron microscope study of Basal Melanocytes and High-Level Clear Cells (Langerhans Cells) in Vitiligo. J Invest Dermatol 1961;37:51-64

5 Gawkrodger DJ, Ormerod AD, Shaw L, et al; Therapy Guidelines and Audit Subcommittee, British Association of Dermatologists; Clinical Standards Department, Royal College of Physicians of London; Cochrane Skin Group; Vitiligo Society. Guideline for the diagnosis and management of vitiligo. Br J Dermatol 2008;159 (05):1051-1076

6 Alikhan A, Felsten LM, Daly M, Petronic-Rosic V. Vitiligo: a comprehensive overview Part I. Introduction, epidemiology, quality of life, diagnosis, differential diagnosis, associations, histopathology, etiology, and work-up. J Am Acad Dermatol 2011;65(03):473-491

7 Savin C. The blood vessels and pigmentary cells of the inner ear. Ann Otol Rhinol Laryngol 1965;74(03):611-622

8 Tosti A, Bardazzi F, Tosti G, Monti L. Audiologic abnormalities in cases of vitiligo. J Am Acad Dermatol 1987;17(2 Pt 1):230-233

9 Shalaby MES, El-Zarea GA, Nassar AI. Auditory function in vitiligo patients. Egypt Dermatol Online J 2006;2(01):1

10 Escalante-Ugalde C, Poblano A, Montes de Oca E, Lagunes R, Saúl A. No evidence of hearing loss in patients with vitiligo. Arch Dermatol 1991;127(08):1240

11 Angrisani RM, Azevedo MF, Pereira LD, Lopes C, Garcia MV. A study on otoacoustic emissions and supression effects in patients with vitiligo. Rev Bras Otorrinolaringol (Engl Ed) 2009;75(01): 111-115

12 Mahdi P, Rouzbahani M, Amali A, Rezaii Khiabanlu S, Kamali M. Audiological manifestations in vitiligo patients. Iran J Otorhinolaryngol 2012;24(66):35-40

13 Sharma L, Bhawan R, Jain RK. Hypoacusis in vitiligo. Indian J Dermatol Venereol Leprol 2004;70(03):162-164

14 Elsaied MA, Naga YAA, Abdo IM. Evaluation of brain stem evoked response in vitiligo patients. J Pan-Arab League Dermatol. 2008; 19:91-97 